van Colen Carl

Ghent University - Marine Biology Research Group

Author(s): Andy Booth ${ }^{1}$, Carl Van Colen ${ }^{2}$,Kaori Sakaguchi-Söder ${ }^{3}$, Paula Sobral $^{4}$, Laura Airoldi $^{5}$, Richard Sempere ${ }^{6}$, Jan Andries Van Franeker ${ }^{7}$, Kerstin Magnusson ${ }^{8}$, Thomas Doyle ${ }^{9}$, Iurgi Salaverria ${ }^{10}$, Dorte Herzke ${ }^{11}$, Amaia Orbea ${ }^{12}$, Geir Wing Gabrielsen ${ }^{13}$, Hartmut Nies ${ }^{14}$, Tamara Galloway $^{15}$, Albert van Oyen ${ }^{16}$

Affiliation(s) : ${ }^{1}$ SINTEF Materials and Chemistry, Trondheim, Norway; ${ }^{2}$ Marine Biology Research Group, Ghent University, Gent, Belgium; ${ }^{3}$ Technical University of Darmstadt, Darmstadt, Germany;

${ }^{4}$ NOVA.ID FCT, Caparica, Portugal; ${ }^{5}$ Alma Mater Studiorum - University of Bologna, Ravenna, Italy; ${ }^{6}$ Aix-Marseille Université, Mediterranean Institute of Oceanography, Marseille, France; ${ }^{7}$ IMARES Wageningen UR, Den Helder, Netherlands; ${ }^{8}$ IVL Swedish Environmental Research Institute, Göteborg, Sweden; ${ }^{9}$ National University of Ireland Galway, Galway, Ireland; ${ }^{10}$ Norwegian University of Science and Technology, Trondheim, Norway; ${ }^{11}$ Norwegian Institute for Air Research, Tromsø, Norway; ${ }^{12}$ Universidad del País Vasco/Euskal Herriko Unibertsitatea, Leioa, Spain; ${ }^{13}$ Norwegian Polar Institute, Tromsø, Norway; ${ }^{14}$ Federal Maritime and Hydrographic Agency, Hamburg, Germany; ${ }^{15}$ University of Exeter, Exeter, United Kingdom; ${ }^{16}$ Control and Research Analysis Thermoplastics, Bocholt, Germany

\title{
PLASTOX: Direct and indirect ecotoxicological impacts of microplastics on marine organisms
}

PLASTOX is one of four consortia under the JPI Oceans Pilot Action "Ecological Aspects of Microplastics" and consists of 15 partners from 11 Member States. The project will investigate the ingestion, foodweb transfer, and ecotoxicological impact of microplastics (MPs), together with persistent organic pollutants (POPs), metals and plastic additive chemicals, on key European marine species and ecosystems. The influence of MP physicochemical properties (size, shape, surface area and composition) on these processes will be evaluated. Laboratory tests and mesocosm studies will be combined with field-based observations and field experiments across a wide range of European marine environments. To bridge the current gap between laboratory assessment using commercially available feedstock MPs and the additive/pollutant-loaded MPs that dominate the marine environment, plastic litter collected from the marine environment will be milled into MPs. Adsorption and desorption behaviour of organic and inorganic pollutants to MPs will be investigated using a range of common POP and metal contaminants, identifying which physicochemical properties are most influential. Uptake through ingestion and other routes will be investigated, and attempts made to quantify MP accumulation in marine organism tissues using state of the art analytical approaches. Acute and sublethal ecotoxicological effects of MPs will be assessed on marine organisms from phyto- and zooplankton to (shell)fish and seabirds, representative of the full range of economically important marine living resources in the EU. PLASTOX will culminate in a series of experiments bringing together the knowledge generated about MPs and POPs/metals to study the combined fate and effects of these marine contaminants in food web studies. 